\title{
A Game Semantics of Local Names and Good Variables
}

\author{
James Laird* \\ Dept. of Informatics, University of Sussex, UK. \\ jiml@sussex.ac.uk
}

\begin{abstract}
We describe a game semantics for local names in a functional setting. It is based on a category of dialogue games acted upon by the automorphism group of the natural numbers; this allows properties of names such as freshness and locality to be characterized semantically. We describe a model of the nu-calculus in this category, and extend it with named references (without bad variables) using names as pointers to a store. After refining the semantics via a notion of garbage collection, we prove that the compact elements are definable as terms, and hence obtain a full abstraction result.
\end{abstract}

\section{Introduction}

Local names are a pervasive and subtle feature of programming languages and other calculi. Not only are they used for manipulating important constructs such as locally bound references and exceptions, name-passing is itself a very expressive computational paradigm, as demonstrated by the $\pi$-calculus, for example. Local names can also represent items of secret information which are dynamically generated, passed between agents and used to access further information or activity. They therefore have a key rôle in specifying properties of secure systems [124.

Game semantics has proved successful in characterizing several features with local names, as demonstrated by fully abstract models of functional languages with locally bound references [234, exceptions [11] and channels [12. However, in one respect, these models do not accurately reflect the way these features are implemented; in the game semantics of references (for example), names are taken to represent storage cells, rather than pointers to storage cells. Symptomatic of the resulting divergence between operational and denotational semantics is the "bad variable" problem; the full abstraction results for these models are contingent upon the presence of objects of reference type which do not behave correctly as storage cells 1 . So the lack of a satisfactory approach to modelling

\footnotetext{
* Supported by EU FET-GC 'MyThS: Models and Types for Security in Mobile Distributed Systems' IST-2001-32617

${ }^{1}$ In [16, McCusker has shown that observational equivalence in Idealized Algol with (active expressions and) bad variables is conservative over observational equivalence in the same language without bad variables. This result highlights how different Ide-
} 
names themselves is a significant limitation when interpreting languages without bad variables such as ML. It would appear to be an even more serious obstacle to giving realistic semantics of languages in which name-passing is fundamental, such as the $\pi$-calculus. In this paper we will describe a category of games in which local names may be interpreted independently of any imperative feature, and use this to construct a model of storage in a language without bad variables in which names are pointers to the store.

In Section 2 we describe a language with local names based on the nu-calculus of Pitts and Stark [20,22]. The latter aims to capture the key features of names in a functional setting, in the absence of associated imperative features such as reference cells. Modelling it may thus be considered a first test for a semantics of local names; existing categories of games, for example, do not offer a natural interpretation of names in isolation in this way. We also extend the nu-calculus with assignment and dereferencing of names with names - i.e. names are used to refer to cells in which names may be stored. The resulting calculus has some of the name-passing capacities of the $\pi$-calculus in a sequential, functional setting. Again, this form of reference has not previously been modelled using standard games techniques.

In Section 3, we describe the basis of our model, a category of (Hyland-Ong style) games and strategies, acted on by the automorphism group of natural numbers, from which we generate an equivalence corresponding to invariance under name substitution. Names are already represented in this way in Stark's model of the nu-calculus in the category of continuous G-sets [22. In the setting of game semantics, of which a key feature is the distinction between Player (representing a program) and Opponent (representing the environment), new issues become evident: which participant in a dialogue introduced a given name, and how can knowledge of it pass from one to the other?

In Section 4 we use our category of games (and constructions for interpreting call-by-value function types developed by Honda and Yoshida [7]) to give a semantics for the nu-calculus. By adding an object corresponding to the store, and using names as pointers to it we are able to give a simple interpretation of the extension with references, Stark's observation 22 that "dynamically created names really do capture the difficult part of ... references; actual value storage is not so hard". We refine our model using a notion of "garbage collection" which exploits the fact that we can make a distinction between globally accessible names (which have been revealed to the environment) and local names (which have not).

In Section 5 we prove (using game semantics techniques such as decomposition and factorization) that all of the compact elements of our "garbage-collected" model are definable as terms, and that contextual equivalence can therefore be characterized semantically, and for finitary terms, decidably. This development is reminiscent of the observation of Jeffrey and Rathke [9, that allowing names to

alized Algol is in this respect from call-by-value languages such as ML (for instance we can distinguish between $\lambda x: \operatorname{var} . x:=0 ; x:=0$ and $\lambda x . x:=0$ in the former, but not the latter). 
leak to the environment through the store simplifies reasoning about contextual equivalence.

\section{$2 \lambda \nu !-$ A Calculus of Names and References}

We will study names and named references via two functional languages with names, the $n u$-calculus [20|22], and an extension with locally named references to local names. The nu-calculus may be described as a simply-typed $\lambda$-calculus with two ground types $o$ (booleans) and $\nu$ (names), extended with various constants: the truth values tt, ff : $o$, a conditional If $: o \rightarrow T \rightarrow T \rightarrow T$ for each type $T$, an equality test on names eq $: \nu \rightarrow \nu \rightarrow o$ and a new-name generator new $: \nu$. The $\lambda \nu$ !-calculus is the nu-calculus extended with constants for writing to and reading from names: assign $: \nu \rightarrow \nu \rightarrow o$ and deref $: \nu \rightarrow \nu$. We use the syntactic sugar $\nu x . M$ for $(\lambda x . M)$ new, $M=N$ for $($ eq $M) N, M:=N$ for $(\operatorname{assign} M) N$ and $! M$ for deref $M$.

Table 1. Operational Semantics of $\lambda \nu$ !

$$
\begin{array}{cc}
\overline{V, \mathcal{E} \Downarrow V, \mathcal{E}} & \frac{n e w,(n, \mathcal{S}) \Downarrow n+1,(n+1, \mathcal{S})}{} \\
\frac{M, \mathcal{E} \Downarrow \lambda x . M^{\prime}, \mathcal{E}^{\prime}}{N, \mathcal{E}^{\prime} \Downarrow V, \mathcal{E}^{\prime \prime} \quad M^{\prime}[V / x], \mathcal{E}^{\prime \prime} \Downarrow U, \mathcal{E}^{\prime \prime \prime}} & M N, \mathcal{E} \Downarrow U, \mathcal{E}^{\prime \prime \prime} \\
\frac{M, \mathcal{E} \Downarrow i, \mathcal{E}^{\prime} \quad N, \mathcal{E}^{\prime} \Downarrow i, \mathcal{E}^{\prime \prime}}{M=N, \mathcal{E} \Downarrow \mathrm{tt}, \mathcal{E}^{\prime \prime}} & \frac{M, \mathcal{E} \Downarrow i, \mathcal{E} \quad N, \mathcal{E}^{\prime} \Downarrow j, \mathcal{E}^{\prime \prime}}{M=N, \mathcal{E} \Downarrow f f, \mathcal{E}^{\prime \prime}} i \neq j \\
\frac{M, \mathcal{E} \Downarrow t \mathrm{t}, \mathcal{E}^{\prime}}{\operatorname{If} M, \mathcal{E} \Downarrow \lambda x y \cdot x, \mathcal{E}^{\prime}} & \frac{M, \mathcal{E} \Downarrow \mathrm{ff}, \mathcal{E}^{\prime}}{\operatorname{If} M, \mathcal{E} \Downarrow \lambda x y \cdot y, \mathcal{E}^{\prime}} \\
\frac{M, \mathcal{E} \Downarrow i, \mathcal{E}^{\prime} \quad N, \mathcal{E}^{\prime} \Downarrow j,(k, \mathcal{S})}{M:=N, \mathcal{E} \Downarrow \mathrm{tt},(k, \mathcal{S}[i \mapsto j])} & \frac{M, \mathcal{E} \Downarrow i,(k, \mathcal{S})}{! M, \mathcal{E} \Downarrow j,(k, \mathcal{S})} \mathcal{S}(i)=j
\end{array}
$$

The operational semantics of $\lambda \nu$ ! subsumes that of the nu-calculus. A program is a closed term of $\lambda \nu$ ! extended with a countable set of names, which are distinguished variables $i: \nu$ for each $1 \leq i<\omega$. We write $k \vdash M$ if no name in $M$ is greater than $k$. A program-in-environment $M, \mathcal{E}$ is a program $M$ together with a pair consisting of a natural number $k$ such that $k \vdash M$, and a store $\mathcal{S}$ (a partial map from $k$ to $k$ ). The "big step" evaluation rules for evaluating a program and an environment to a value (a $\lambda$-abstraction, name or truth value) and an environment are shown in Table 1 . Note that unlike the nu-calculus, failure to converge to a value is possible, i.e. if a reference is dereferenced before any value has been assigned to it.

We adopt a standard definition of contextual equivalence: given terms $M, N$ : $T$ (of a given language $\mathcal{L}$ ), $M \approx_{T} N$ in $\mathcal{L}$ if for all closing contexts $C$ [_] : o of $\mathcal{L}$, 
$C[M] \Downarrow$ tt if and only if $C[N] \Downarrow \mathrm{tt}$. (Where $M \Downarrow V$ if $M,(0, \perp) \Downarrow V, \mathcal{E}$ for some $\mathcal{E}$.

\section{Games with Names}

Our notion of game is based on the dialogue games of Hyland and Ong [8] (and Nickau [18]), to which we add structure for manipulating a countable set of names, in the form of an action of the automorphism group of the natural numbers. This generates an equivalence on strategies corresponding to invariance under substitution of names2. We give brief (and slightly non-standard) definitions of arenas and legal sequences and refer the reader to the literature ([8], 10:7] etcetera) for more detailed explanation.

An (underlying) arena $A$ is a tuple $\left(M_{A}, M_{A}^{I}, \lambda_{A}, \vdash_{A}\right)$ consisting of a set of moves $M_{A}$, a subset $M_{A}^{I} \subseteq M_{A}$ of initial moves, a question/answer labelling $\lambda_{A}: M_{A} \rightarrow\{Q, A\}$, and an enabling relation $\vdash_{A} \subseteq M_{A} \times\left(M_{A}-M_{A}^{I}\right)$ such that no answer is enabled by an answer. We require that the enabling relation partitions the set of moves according to the following rule: every initial move is an Opponent move, and every move enabled by an Opponent move is a Player move, and vice-versa. We describe an arena as $A$-rooted if all of its initial moves are answers.

A justified sequence over the arena $A$ is a sequence of moves of $A$ together with a pointer from each non-initial move to an enabling move. The set $L_{A}$ of legal sequences of $A$ consists of the finite justified sequences which are well-opened (contain at most one initial move), alternating (Opponent moves are followed by Player moves and vice-versa), well-bracketed (every answer is justified by the last-asked open question) and satisfy the visibility condition (the Player and Opponent views 815] of every subsequence are justified sequences).

Let $G$ be the topological group of natural number automorphisms with the product topology on $\mathbb{N}^{\mathbb{N}}$, for which a basis of neighborhoods of the identity is $\left\{\operatorname{stab}_{G}(k) \mid k \subseteq\right.$ fin $\left.\mathbb{N}\right\}[22$. By a continuous action of $G$ upon a set $A$, we will mean a $G$-action which is continuous with respect to the discrete topology on $A$. So the stabiliser of any element $a \in A$ is open in $G$ and thus equal to the stabiliser of a finite subset $k \subseteq \mathbb{N}$, the support of $a$.

Definition 1. A $\nu$-arena is an arena $A$ and a continuous action of $G$ on $M_{A}$ such that $\lambda_{A}(\pi(m))=\lambda_{A}(m)$ and $m \vdash n$ iff $\pi(m) \vdash \pi(n)$.

The group action extends naturally to legal sequences.

Lemma 1. If $A$ is a $\nu$-arena, then there is a continuous action of $G$ on $L_{A}$ defined $\pi\left(m_{1} m_{2} \ldots m_{n}\right)=\pi\left(m_{1}\right) \pi\left(m_{2}\right) \ldots \pi\left(m_{n}\right)$.

\footnotetext{
${ }^{2}$ A similar notion of equivalence is used in [5], also to preserve parametricity in games with a countable set of labels. The key difference is that in the model of $\lambda \nu$ ! names can be passed between strategies, and so equivalence cannot be described componentwise as in [5].
} 
We write $\nu(s)$ for the support of $s$, and use it to define associated functions $P_{\nu}, O_{\nu}$ which identify the sets of new names introduced by Player (or by complementation, Opponent) in a legal sequence.

Definition 2. The functions $P_{\nu}, O_{\nu}$ from legal sequences to $\mathcal{P}_{\text {fin }}(\mathbb{N})$ are defined: $P_{\nu}(\varepsilon)=\varnothing$,

$P_{\nu}(s a)=P_{\nu}(s) \cup(\nu(s a)-\nu(s))$ if a is Player move,

$P_{\nu}(s a)=P_{\nu}(s)$ otherwise.

$O_{\nu}(s)=\nu(s)-P_{\nu}(s)$.

We write $\sim$ for the equivalence relation on legal sequences determined by the orbits of the group action - i.e. $s \sim t$ if $\exists \pi \in G . \pi(s)=t$. A strategy on a $\nu$-arena is, in essence, the orbit of a deterministic strategy on the underlying arena.

Definition 3. Let $A$ be a $\nu$-arena. A $\nu$-strategy $\sigma: A$ is a non-empty and evenprefix-closed set of even-length legal sequences of $A$ subject to the following conditions:

- If $s \in \sigma$ and $s \sim t$ then $t \in \sigma$.

- If $s a, t b \in \sigma$ and $s \sim t$, then $s a \sim t b$.

\subsection{A Call-by-Value Category of Games}

We will now construct a premonoidal category of games in which to model the call-by-value $\lambda$-calculus aspect of $\lambda \nu$ !. We follow essentially the constructions of Honda and Yoshida [7] or variants described by Laurent [13. In each case the group action on compound arenas is defined pointwise. The key novelty thus lies in the definition of composition of strategies, since we must maintain distinctness of fresh names.

Definition 4. Given $\nu$-arenas $A_{1}, A_{2}$ we define a (Q-rooted) $\nu$-arena $A_{1} \rightarrow A_{2}$ :

$-M_{A_{1} \rightarrow A_{2}}=M_{A_{1}}+M_{A_{2}}$,

$-\lambda_{A_{1} \rightarrow A_{2}}\left(\operatorname{in}_{\mathrm{i}}(\mathrm{m})\right)=\mathrm{Q}$, if $i=1$ and $m \in M_{A_{1}}^{I}$,

$\lambda_{A_{1} \rightarrow A_{2}}\left(\mathrm{in}_{\mathrm{i}}(\mathrm{m})\right)=\lambda_{\mathrm{A}_{\mathrm{i}}}(\mathrm{m})$, otherwise,

$-M_{A_{1} \rightarrow A_{2}}^{I}=\operatorname{in}_{1}\left(\mathrm{M}_{\mathrm{A}_{1}}\right)$,

$-\vdash_{A_{1} \rightarrow A_{2}}=\left[\vdash_{A_{1}}, \vdash_{A_{2}}\right] \cup\left(\operatorname{in}_{1}\left(\mathrm{M}_{\mathrm{A}_{1}}^{\mathrm{I}}\right) \times \operatorname{in}_{2}\left(\mathrm{M}_{\mathrm{A}_{2}}^{\mathrm{I}}\right)\right)$

$-\pi\left(\operatorname{in}_{\mathrm{i}}(\mathrm{m})\right)=\operatorname{in}_{\mathrm{i}}(\pi(\mathrm{m}))$.

So the initial moves in $A_{1} \rightarrow A_{2}$ are the initial moves from $A_{1}$ relabelled as questions (to which the initial moves from $A_{2}$ are the answers).

Composition of strategies $\sigma: A \rightarrow B$ and $\tau: B \rightarrow C$ is, as usual, by allowing interaction in $B$ which is then hidden. However, further conditions are required to ensure that the new names introduced by $\sigma$ are disjoint from those introduced by $\tau$, and from those introduced by Opponent in $C$ (and vice-versa).

Definition 5. The set of well-formed interaction sequences $I_{A, B, C}$ is the set of legal sequences $s \in L_{(A \rightarrow B) \rightarrow C}$ which satisfy the follow conditions:

$-P_{\nu}\left(s\lceil A \rightarrow B) \cap P_{\nu}(s\lceil B \rightarrow C)=\varnothing\right.$, 
$-\left(P_{\nu}(s \uparrow A \rightarrow B) \cup P_{\nu}(s \mid B \rightarrow C)\right) \cap O_{\nu}(s \mid A \rightarrow C)=\varnothing$.

Given $\sigma: A \rightarrow B, \tau: B \rightarrow C, \sigma \mid \tau=\left\{s \in I_{A, B, C}|s| A \rightarrow B \in \sigma \wedge s\lceil B \rightarrow C \in \tau\}\right.$ and $\sigma ; \tau: A \rightarrow C=\left\{s \in L_{A \rightarrow C} \mid \exists t \in I_{(A \rightarrow B) \rightarrow C} . s=t\lceil A \rightarrow C\}\right.$.

To establish that we may define a category based on this notion of composition, it is necessary prove that it yields a well-defined strategy (note that this would not be the case without the fresh name restrictions on interaction sequences) and is associative.

Lemma 2. The composition of $\nu$-strategies is a well-defined $\nu$-strategy.

Proof. We need to show that if $s \in \sigma ; \tau$ and $s \sim t$, then $t \in \sigma ; \tau$ and if $s a, t b \in \sigma ; \tau$ and $s \sim t$ then $s a \sim t b$. The first part is straightforward, since if $s \in \sigma \mid \tau$ and $\pi(s\lceil A \rightarrow C)=t$, then $\pi(s) \in \sigma \mid \tau$ and $\pi(s)\lceil A \rightarrow C=t$.

To establish the second property, we prove by induction on sequence length that if $s a, t b \in \sigma \mid \tau$ and $s \uparrow A \rightarrow C \sim t \uparrow A \rightarrow C$, then $s a \sim t b$ and hence $s a \uparrow A \rightarrow C \sim t b\lceil A \rightarrow C$. The proof uses the freshness assumptions on interaction sequences to show that when $\sigma$ introduces a new name in $s \uparrow A \rightarrow B$ then it is genuinely new in $s$ (and similarly for $\tau$ ) and also that when Opponent introduces a new name in $s\lceil A \rightarrow C$ then it is new in $s$.

Lemma 3. Composition of $\nu$-strategies is associative.

Proof. This follows the proof of associativity for ordinary strategies [10] - we show that given $\rho: A \rightarrow B, \sigma: B \rightarrow C, \tau: C \rightarrow D$, if $s \in \rho ;(\sigma ; \tau)$ then there exists $t \in L_{A \rightarrow B \rightarrow C \rightarrow D}$ such that $t \uparrow(A \rightarrow B) \rightarrow C \in \rho|\sigma, t \uparrow(B \rightarrow C) \rightarrow D \in \sigma| \tau$ and $s=t\lceil A, D$ and hence $s \in(\rho ; \sigma) ; \tau$. The complicating factor in the case of $\nu$-strategies is that there may be names introduced in the hidden components $B$ and $C$ which violate the freshness assumptions for interaction sequences - we use the saturation of strategies with respect to $\sim$ and the fact that every move has finite support to show that we can always make choices of fresh names which avoid this.

Since any copycat strategy never introduces new names, it is straightforward to show that for each arena the standard notion of identity strategy is well-defined and has the required properties.

Proposition 1. The A-rooted $\nu$-arenas form a category $\nu \mathcal{G}$ in which morphisms from $A$ to $B$ are $\nu$-strategies on $A \rightarrow B$.

We now define premonoidal structure on $\nu \mathcal{G}$ which is essentially the same as that described in [7, but with restrictions on the sharing of names.

Definition 6. From $A$-rooted arenas $A_{1}, A_{2}$, form an $A$-rooted arena $A_{1} \odot A_{2}$ :

$-M_{A_{1} \odot A_{2}}=\left(M_{A_{1}} \times M_{A_{2}}^{I}\right) \cup\left(M_{A_{1}}^{I} \times M_{A_{2}}\right)$

$-\lambda_{A_{1} \odot A_{2}}\left(\left\langle m_{1}, m_{2}\right\rangle\right)=\lambda_{A_{2}}\left(m_{2}\right)$, if $m_{1} \in M_{A_{1}}^{I}$,

$\lambda_{A_{1} \odot A_{2}}\left(\left\langle m_{1}, m_{2}\right\rangle\right)=\lambda_{A_{1}}\left(m_{1}\right)$, otherwise,

- $M_{A_{1} \odot A_{2}}^{I}=M_{A_{1}}^{I} \times M_{A_{2}}^{I}$, 
$-\vdash_{A_{1} \odot A_{2}}=\left(\vdash_{A_{1}} \otimes \mathrm{Id}\right) \cup\left(\mathrm{Id} \otimes \vdash_{A_{2}}\right)$.

$-\pi(\langle m, n\rangle)=\langle\pi(m), \pi(n)\rangle$,

The unit I for $\odot$ is the A-rooted arena with a single (initial) move.

Given a legal sequence $t \in L_{A_{1} \odot A_{2}}$, we obtain a legal sequence $t \uparrow A_{i} \in L_{A_{i}}$ by taking the $i$ th projection from each move, and then erasing all initial moves except the first from the result.

Proposition 2. $(\nu \mathcal{G}, I, \odot)$ is a symmetric premonoidal category.

Proof. For each object $A$, we define endofunctors $A \odot_{-}$and _ $\odot A: \nu \mathcal{G} \rightarrow \nu \mathcal{G}$ : given $\sigma: B \rightarrow C$, let $A \odot \sigma: A \odot B \rightarrow A \odot C=$

$$
\left\{s \in L_{A \odot B \rightarrow A \odot C}|s| B \rightarrow C \in \sigma \wedge s \mid A \rightarrow A \in \operatorname{id}_{A} \wedge P_{\nu}(s \mid B \rightarrow C)=P_{\nu}(s)\right\} .
$$

We now identify, via conditions on strategies, a subcategory of $\nu \mathcal{G}$ for which the premonoidal product is cartesian. The first condition is totality, as used in [7].

Definition 7. A morphism $f: A \rightarrow B$ is total if $g$; $f=\perp$ implies $g=\perp$ (where $\perp$ is the empty strategy). So $\sigma: A \rightarrow B$ is total if it responds to the initial question in $A$ with the initial answer in $B$. A sequence in which this occurs is said to be total.

Our further condition on total strategies is essentially thread independence [4] up to $\sim$, plus conditions on the introduction and sharing of names across threads.

Definition 8. The thread of a total sequence qasb $\in L_{A \rightarrow B}$ is a legal sequence of $A \rightarrow B$ defined as follows:

thread $(q a s b)=q a b$ if $b$ is justified by $a$, $\operatorname{thread}($ qasb $)=\operatorname{thread}($ qas $)$ b otherwise.

Definition 9. A total sequence qas $\in L_{A \rightarrow B}$ is thread-independent with respect to names if:

- Player does not introduce any new names with the move a - i.e $P_{\nu}(q a)=\varnothing$.

- Whenever Player introduces a name which is fresh in its thread, it is fresh in qas - i.e. if qatb 巨even qas then $(\nu(\operatorname{thread}($ qatb $))-\nu(\operatorname{thread}(q a t))) \cap \nu(q a t)=$ $\varnothing$.

A total strategy $\sigma$ is thread-independent if each sequence in $\sigma$ is threadindependent with respect to names and whenever $s, t a \in \sigma$ and $\operatorname{thread}(s b) \sim$ thread $(t)$ then there exists $s b c \in \sigma$ such that thread $(s b c) \sim \operatorname{thread}(t a)$.

Lemma 4. The thread-independent strategies form a subcategory $\nu \mathcal{G}_{t}$ of $\nu \mathcal{G}$ upon which $\odot$ is a cartesian product.

Proof. Compositionality of thread-independent strategies is straightforward. We also observe that _ $\odot_{\text {_ }}$ is a symmetric monoidal product on the total strategies, as in [7] (and $I$ is a terminal object in this category). Further, we have copycat strategies yielding natural maps $\pi_{i}: A_{1} \odot A_{2} \rightarrow A_{i}$ and $\delta: A \rightarrow A \odot A$ such that $\delta ; \pi_{i}=\operatorname{id}_{A}, \delta_{A_{1} \odot A_{2}} ; \pi_{1} \odot \pi_{2}=\operatorname{id}_{A_{1} \odot A_{2}}$ and if $f: A \rightarrow B$ is total and thread-independent then $\delta_{A} ;(f \odot f)=f ; \delta_{B}$. 
Thus we have a Freyd category [21]; a cartesian category $\nu \mathcal{G}_{t}$, a symmetric premonoidal category $\nu \mathcal{G}$, and an identity-on-objects strict symmetric premonoidal functor (inclusion) from $\nu \mathcal{G}_{t}$ to $\nu \mathcal{G}$. Moreover, this is a closed Freyd category.

Proposition 3. Inc(_) $\odot A: \nu \mathcal{G}_{t}: \rightarrow \nu \mathcal{G}$ has a right adjoint $A \rightarrow_{-}: \nu \mathcal{G} \rightarrow \nu \mathcal{G}_{t}$.

Proof. Given a $Q$-rooted arena $B$, let $\uparrow B$ be the $A$-rooted arena obtained by adding to $B$ a single initial answer (invariant under $G$ action) which enables all of the initial moves of $B$. We define $A \rightarrow B=\uparrow(A \rightarrow B)$.

We have a one-to-one correspondence $\phi$ between legal sequences on $A \odot B \rightarrow C$ (which have the form $\langle m, n\rangle \cdot s$ ) and total sequences on $A \rightarrow(B \rightarrow C$ ) (which have the form $\operatorname{man} \cdot s)$. This gives rise to the required natural isomorphism between $\nu \mathcal{G}(A \odot B, C)$ and $\nu \mathcal{G}_{t}(A, B \rightarrow C)$ : given $\sigma: A \odot B \rightarrow C$, let $\Lambda(\sigma): A \rightarrow B \rightarrow C$ be the least set of legal sequences such that $\varepsilon, q a \in \Lambda(\sigma)$, and if $s \in \Lambda(\sigma)$, sab is thread-independent with respect to names and $\phi(\operatorname{thread}(s a b)) \in \sigma$ then $s a b \in \sigma$.

\section{Semantics of $\lambda \nu$ !}

In [23], Stark describes a notion of categorical model for the nu-calculus. It is based on monadic models of the computational $\lambda$-calculus, but transfers readily to the more direct description used here. (Note that $\nu \mathcal{G}$ is equivalent to the Kleisli category of the strong monad $\mathbf{T} A=I \rightarrow A$ on $\nu \mathcal{G}_{t}$.) The categorical properties required in 23] to interpret the nu-calculus may be summarised as follows:

- To interpret the call-by-value $\lambda$-calculus, a sound model of Moggi's computational metalanguage.

- To interpret the type o of booleans, a disjoint coproduct of the terminal object $\left(\right.$ of $\nu \mathcal{G}_{t}$ ) with itself $-\nu \mathcal{G}$ in fact has all small coproducts, obtained by taking the disjoint sum of arenas 13, thus $I+I$ is the arena with two distinct initial answer moves, invariant under $G$-action.

- To interpret the type $\nu$ of names, a distinguished decidable object $N$ - in $\nu \mathcal{G}$ this is the $\nu$-arena with a set of initial answer-moves indexed over $\mathbb{N}$, which are acted on according to their indices: $M_{N}=M_{N}^{I}=\left\{m_{i} \mid i \in \mathbb{N}\right\}$, $\lambda\left(m_{i}\right)=A$ and $\pi\left(m_{i}\right)=m_{\pi(i)}$ for each $i$. The map eq $: N \odot N \rightarrow I+I$ which completes the pullback square $t_{N} ; \mathrm{in}_{1}: \mathrm{N} \rightarrow \mathrm{I}+\mathrm{I}=\delta$; eq and with which we interpret the equality test is the strategy $\left\{\left\langle m_{i}, m_{i}\right\rangle \cdot \mathrm{tt} \mid i \in \mathbb{N}\right\} \cup$ $\left\{\left\langle m_{i}, m_{j}\right\rangle \cdot \mathrm{ff} \mid i, j \in \mathbb{N} \wedge i \neq j\right\}$.

- To interpret the new-name declaration new : $o$, a distinguished map new : $I \rightarrow N$. This is the total strategy which responds to the initial question with any move in $N$ - i.e. new $=\left\{q m_{i} \mid i \in \mathbb{N}\right\}$. It is straightforward to verify that new satisfies further equations given in 23. stipulating that new names are distinct from all others, the order in which they are generated is not relevant, and that unused names may be ignored.

Proposition 4. $\nu \mathcal{G}$ is a categorical model of the nu-calculus in the sense of [23]. 
As examples we consider two contextual equivalences of the nu-calculus described by Pitts and Stark [22. The first is $\nu x . \lambda y . x=y \approx_{\nu \rightarrow o} \lambda y$.ff. The term $\nu x \cdot \lambda y \cdot x=y: \nu \rightarrow o$ is interpreted by composing new $: I \rightarrow N$ with $\llbracket x: \nu \vdash \lambda y \cdot x=y \rrbracket: N \rightarrow(N \rightarrow I+I)=\Lambda($ eq $)=\left\{m_{i} a m_{i}\right.$ tt $\mid i \in$ $\mathbb{N}\} \cup\left\{m_{i} a m_{j} \mathrm{ff} \mid i, j \in \mathbb{N} \wedge i \neq j\right\}$. By the freshness assumption for composition the name supplied by new must be distinct from the name supplied by Opponent in $(N \rightarrow I+I)$ and so $\llbracket \nu x \cdot \lambda y \cdot x=y \rrbracket=\left\{q a m_{i}\right.$ ff $\left.\mid i \in \mathbb{N}\right\}=\llbracket \lambda y$.ff $\rrbracket$. (However, these terms do not have the same denotation in the functor category model 22.)

As a second example, we consider the terms $\lambda f: \nu \rightarrow$ o.tt and $\nu x . \nu y . \lambda f$ : $\nu \rightarrow o$. $(f x=f y)$, which are contextually equivalent in the nu-calculus, but are not denotationally equivalent in our model. In the former, Player supplies the value tt without querying the agument, in the latter, Player queries the argument twice, supplying it with distinct names on each occasion (see Figure 1). They can be distinguished in $\lambda \nu$ !, for example by the context $\nu z . z:=z ;([\cdot](\lambda n . z:=$ $n)) ; ! z=z$ which returns tt in the first case and ff in the second.

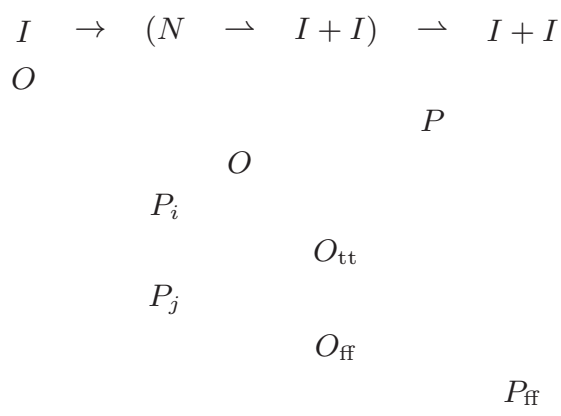

Fig. 1. A typical play in $\llbracket \nu x . \nu y \cdot \lambda f .(f x=f y) \rrbracket$.

\subsection{Semantics of the Store}

Our first attempt to give a games model of $\lambda \nu$ ! illustrates Stark's observation [22] that having given a correct interpretation of local names, it is quite straightforward to use them to store values. We extend our semantics of the nu-calculus to $\lambda \nu$ ! by simply adding an object $S$ - an arena representing the store - to the contexts in which terms are interpreted. That is, we interpret $\Gamma \vdash M: T$ as a morphism from $\llbracket \Gamma \rrbracket \odot S$ to $\llbracket T \rrbracket$. For general reasons this is still a sound model of the nu-calculus.

Definition 10. For any object $S$, the co-Kleisli category $\nu \mathcal{G}_{s}$ of the co-monad _ $\odot S$ (with triple $\left(\_\odot S, \pi_{l}\right.$, assoc; $\left.\left.\delta\right)\right)$ is a closed Freyd category and a model of the nu-calculus. 
To interpret assignment and dereferencing we adapt the approach used in previous games models of state [2]4; we fix the store object $S$ to be the product of its "write" and "read" methods: $S=(N \odot N \rightarrow I) \odot(N \rightarrow N))^{3}$. We define assignment and dereferencing simply in terms of projections from this product:

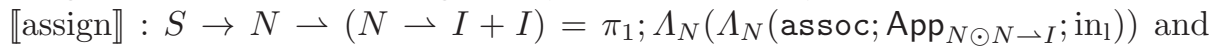
«deref』: $S \rightarrow N \rightarrow N=\pi_{2}$. We shall label the non-initial moves in the "write" component $N \odot N \rightarrow I$ as $\{\operatorname{write}(i, j) \mid i, j \in \mathbb{N}\}$ (Player moves) and ok (Opponent moves), and the non-initial moves in the "read" component $(N \rightarrow N)$ as $\{\operatorname{read}(i) \mid i \in \mathbb{N}\}$ (Player moves) and $\{\operatorname{return}(j) \mid j \in \mathbb{N}\}$ (Opponent moves).

To determine the behaviour of a program under evaluation we compose it with a total strategy store $: I \rightarrow S$, which responds to write $(i, j)$ with ok and to $\operatorname{read}(i)$ with return $(j)$, where $j$ is the last value to have been written to $i$, if any.

Given a store $\mathcal{S}$, we define the $\lambda \nu$ !-term $\operatorname{ass}(i, \mathcal{S}): o$ to be $i:=j$ if $\mathcal{S}(i)=j$ and tt if $\mathcal{S}(i) \uparrow$. For a program-in-environment $M,(k, \mathcal{S})$, we define $\llbracket M,(k, \mathcal{S}) \rrbracket$ : $I \rightarrow \llbracket T \rrbracket=\left(\right.$ new $^{k} \odot$ store $) ; \llbracket \operatorname{ass}(1, \mathcal{S}) ; \ldots ; \operatorname{ass}(k, \mathcal{S}) ; M \rrbracket$.

Lemma 5. The following equations hold:

$-\llbracket V(i:=j),(k, \mathcal{S}) \rrbracket=\llbracket V \mathrm{tt},(k, \mathcal{S}[i \mapsto j] \rrbracket$,

- $V V ! i,(k, \mathcal{S}) \rrbracket=\llbracket V j,(k, \mathcal{S}) \rrbracket(\mathcal{S}(i)=j)$.

Proof. This is by analysis of the interaction between programs and the store strategy.

Proposition 5. $M,(k, \mathcal{S}) \Downarrow V,\left(k^{\prime}, \mathcal{S}^{\prime}\right)$ if and only if for any program $\lambda x . N$ such that $k \vdash \lambda x . N, \llbracket(\lambda x . N M),(k, \mathcal{S}) \rrbracket=\llbracket N[V / x],\left(k^{\prime}, \mathcal{S}^{\prime}\right) \rrbracket$.

Proof. Soundness is established by induction on evaluation, using the equations established in Lemma 5 and the properties of $\nu \mathcal{G}$ as a model of the nu-calculus. Adequacy is proved using a standard reducibility predicate argument.

Corollary 1. For any closed $M: o, M \Downarrow$ tt if and only if store; $\llbracket M \rrbracket=\llbracket \mathrm{tt} \rrbracket$.

\subsection{Garbage Collection}

An immediately apparent feature of our model of $\lambda \nu$ ! is that interaction with the store always remains a visible part of each strategy, even when it takes place in a cell which has a private name which is never revealed to the environment. This leads to some particularly egregious failures of full abstraction; the denotations of $\nu x . x:=x$ and tt are distinct, for example. For the same reason our model also lacks the finite definability property. That is, not all of the compact strategies on type-objects are denotations.

We shall solve these problems by refining our semantics via a notion of garbage collection - hiding parts of the store which are never globally accessible. We define the latter property by stipulating that a name is globally accessible if has been revealed outside the store, or in a location which is already globally accessible.

${ }^{3} S$ corresponds to a $\mathbb{N}$-indexed collection of copies of a "cell" object $C=(I-$ $N) \odot(N \rightarrow I)[2$, to which names act as pointers. 
Definition 11. The set of global names of the sequence $s \in L_{A \odot S \rightarrow B}$ is defined: $\operatorname{glob}(\varepsilon)=\varnothing$, $\operatorname{glob}(s a)=\operatorname{glob}(s) \cup \nu(a)$, if a is a move in $A$ or $B$, $\operatorname{glob}(s w r i t e(i, j))=\operatorname{glob}(s) \cup\{j\}$, if $i \in \operatorname{glob}(s)$, $\operatorname{glob}(s a)=\operatorname{glob}(s)$, otherwise.

We impose an associated condition on legal sequences, requiring that Opponent can only use names which are either new or already globally accessible.

Definition 12. A sequence $s \in L_{A \odot S \rightarrow B}$ satisfies the locality requirement if for every ta $\sqsubseteq_{\text {odd }} s, \nu(a) \subseteq(\nu(t a)-\nu(t)) \cup \operatorname{glob}(t)$.

The garbage collection of a strategy $\sigma: A \odot S \rightarrow B$ is obtained by hiding interaction with the store strategy in those cells in $S$ which are local in $\sigma$ (a cell is local if its name never becomes globally accesible).

Definition 13. Given a strategy $\sigma$ and $s a \sqsubseteq t \in \sigma$, we say that a is local in $\sigma$ if it has the form write $(i, j)$ or $\operatorname{read}(i)$, or is enabled by such a move, and there is no $t^{\prime} \in \sigma$ (satisfying the locality requirement) such that $s a \sqsubseteq t^{\prime}$ and $i \in \operatorname{glob}\left(t^{\prime}\right)$.

For each $s \in \sigma$, let $s \backslash \operatorname{loc}(\sigma)$ be the result of erasing all of the occurrences of moves from $s$ which are local in $\sigma$, and let $s \mid \operatorname{loc}(\sigma)$ be the result of erasing all of the moves in $s$ which are not local in $\sigma$. We define the garbage collection of $\sigma$ :

$$
\gamma(\sigma)=\left\{t \in L_{A \odot S \rightarrow B} \mid \exists s \in \sigma . q a \cdot(s \mid \operatorname{loc}(\sigma)) \in \text { store } \wedge s \backslash \operatorname{loc}(\sigma)=t\right\}
$$

Lemma 6. If $\sigma: A \odot S \rightarrow B$ then $\gamma(\sigma)$ is a well defined strategy on $A \odot S \rightarrow B$.

Proof. We show by induction on sequence length that if $s a, t b \in \gamma(\sigma)$, and $s \sim t$, then there exists $s^{\prime} a, t^{\prime} b \in \sigma$ such that $s^{\prime} \sim t^{\prime}$ and $s=s^{\prime} \backslash \operatorname{loc}(\sigma)$ and $t=t^{\prime} \backslash \operatorname{loc}(\sigma)$ and hence $s a \sim t b$.

Lemma 7. For strategies $\sigma: A \rightarrow B, \tau: B \rightarrow C, \gamma(\sigma ; \tau)=\gamma(\gamma(\sigma) ; \gamma(\tau))$.

Proof. We consider interaction sequences $s$ in $\sigma^{\dagger} \mid \tau \subseteq I_{A \odot S, B \odot S, C}$ (where $\sigma^{\dagger}$ : $A \odot S \rightarrow B \odot S=$ assoc; $(A \odot \delta) ;(\sigma \odot S))$. Note that the freshness assumptions on interaction sequences imply that if Opponent obeys the locality condition in $s\lceil A \odot S \rightarrow B$ and $s\lceil B \odot S \rightarrow C$ then Opponent obeys the locality condition in $s \uparrow A \odot S \rightarrow C$. We show that if we erase moves (in both copies of the store) which are local with respect to the whole interaction sequence (hence obtaining an interaction sequence in $\gamma(\sigma)^{\dagger} \mid \gamma(\tau)$ ), and then restrict to $A \odot S \rightarrow C$ and garbage collect, then this is equivalent to restricting $s$ to $A \odot S \rightarrow C$ and garbage collecting.

A strategy $\sigma$ is said to be garbage-collected if $\gamma(\sigma)=\sigma$ - i.e $\sigma$ has no local names. We define a category $\gamma \nu \mathcal{G}$ of garbage-collected strategies, in which the composition of $\sigma$ and $\tau$ is defined to be $\gamma(\sigma ; \tau)$. Lemma 7 is used to establish that this is associative, and that $\gamma$ acts as an (identity on objects) functor from $\nu \mathcal{G}$ to $\gamma \nu \mathcal{G}$. From the soundness result for the original model (Proposition 5]) we obtain the following. 
Proposition 6. $M \Downarrow$ tt if and only if $\llbracket M \rrbracket_{\gamma \nu \mathcal{G}}=\gamma\left(\llbracket M \rrbracket_{\nu \mathcal{G}}\right)=\llbracket \mathrm{tt} \rrbracket$.

If $T$ is a $\nu$-free type, then every strategy $\sigma: S \rightarrow \llbracket T \rrbracket$ has no globally accessible names, and therefore $\gamma(\sigma)$ contains no interaction with the store. So for these types, our model is equivalent to (the boolean fragment of) Abramsky and McCusker's model of RML.

\section{Definability and Full Abstraction}

To prove definability of compact strategies in the garbage-collected model, we combine two standard proof techniques; factorization of strategies into the composition of a finitary innocent strategy with a series of copies of the new strategy and the store, and a proof by decomposition that the innocent strategies are definable in $\lambda \nu !-\{$ new $\}$. Note first that if $\sigma: S \rightarrow \llbracket T \rrbracket$ is compact with respect to the inclusion order then both the the length of sequences in $\sigma$, and the number of names introduced in each sequence is bounded. Recall that a strategy $\sigma$ is deterministic if $s a, s b \in \sigma$ implies $a=b$. Note that a $\nu$-strategy is deterministic if and only if it never introduces any new names - i.e. for all $s \in \sigma, P_{\nu}(s)=\varnothing$.

Lemma 8. Given any compact strategy $\sigma: A \rightarrow B$, there exists a deterministic strategy $\widehat{\sigma}: N^{k} \odot A \rightarrow B$ such that $\left((\text { new })^{k} \odot \mathrm{id}_{A}\right) ; \widehat{\sigma}=\sigma$.

Proof. Opponent's first move in $A \odot N^{k} \rightarrow B$ supplies names $n_{1}, \ldots, n_{k} . \widehat{\sigma}$ then plays as $\sigma$ except that where $\sigma$ uses a Player-introduced name, $\widehat{\sigma}$ copies one of the $n_{1}, \ldots, n_{k}$.

We now use a further factorization to reduce the deterministic strategies to finitary innocent strategies composed with multiple copies of a strategy cell : $I \rightarrow(N \rightarrow I+I) \odot(I+I \rightarrow N)$. (A strategy is innocent if its behaviour is always determined by the Player view [8 15], an innocent strategy is finitary if its set of views is finite.) The cell strategy corresponds to (a lifting of) the cell strategy defined in [2], in the "read component" $N \rightarrow I+I$ it returns the last value (if any) written to the "write" component $N \rightarrow I+I$.

Lemma 9. Let $\sigma: A \rightarrow B$ be a deterministic compact strategy. Then there exists $n \in \mathbb{N}$, and a finitary innocent strategy $\widetilde{\sigma}:((N \rightarrow I+I) \odot(I+I \rightarrow$ $N))^{m+1} \odot N^{n} \odot A \rightarrow B$ such that $\sigma=$ cell $^{m+1} \odot$ new $^{k} \odot \operatorname{id}_{A} ; \tilde{\sigma}$.

Proof. The proof follows the factorization of a "knowing" strategy into the composition of an innocent strategy and a cell described by Abramsky and McCusker [2]. $\widetilde{\sigma}$ uses one copy of $(N \rightarrow I+I) \odot(I+I \rightarrow N)$ as a reference cell to record the history of play in $A$ and $B$, via natural number encoding (we use the additional names supplied by Opponent to represent the numbers $1, \ldots, n$ ). We require the additional reference cells to store the new names introduced by Opponent in the course of play, as these cannot be encoded.

Finally, we prove definability of the finitary innocent strategies. 
Lemma 10. Given a finitary innocent strategy $\sigma: S \rightarrow \llbracket T \rrbracket$, there exists a term $M_{\sigma}: T$ of $\lambda \nu !-\{$ new $\}$ such that $\llbracket M_{\sigma} \rrbracket=\sigma$.

Proof. We prove this using a decomposition of innocent strategies which closely follows Honda and Yoshida's proof of finite definability for their model of callby-value PCF [7. The only difference is at the level of first-order functions on names, which can compare and copy their inputs, but may not perform any other arithmetic operations on them.

Proposition 7. Every compact strategy on a $\lambda \nu$ !-type object is definable as a term of $\lambda \nu !$.

Proof. Given a strategy $\sigma: S \rightarrow \llbracket T \rrbracket$, we apply Lemmas 8 and 9 to obtain an innocent strategy $\sigma^{\prime}:((N \rightarrow I+I) \odot(I+I \rightarrow N))^{m+1} \odot N^{k+n} \odot S \rightarrow \llbracket T \rrbracket$ such that $\sigma=$ cell $^{m+1} \odot$ new $^{k+n} \odot \mathrm{id}_{S} ; \sigma^{\prime}$. By Lemma 10, $\sigma^{\prime}$ is definable as a term $y_{1}$ : $\nu \rightarrow o, z_{1}: o \rightarrow \nu, \ldots, y_{m+1}: \nu \rightarrow o, z_{m+1}: o \rightarrow \nu, x_{1}: \nu, \ldots, x_{n+k}: \nu \vdash M_{\sigma^{\prime}}: T$. Thus we define $M_{\sigma}=$

$\nu x_{1} \ldots x_{n+k} \cdot \nu w_{1} \ldots w_{m+1} \cdot\left(\left(\left(\lambda y_{1} \ldots y_{m+1} \cdot \lambda z_{1} \ldots z_{m+1} \cdot M_{\sigma^{\prime}}\right) \boldsymbol{\lambda} \boldsymbol{u} \cdot \boldsymbol{w}:=\boldsymbol{u}\right) \boldsymbol{\lambda} \boldsymbol{v} \cdot ! \boldsymbol{w}\right)$

By a standard argument 8,5, we may now show that observational equivalence in $\lambda \nu$ ! corresponds to the intrinsic equivalence $\simeq$ on the model, where $f: A \rightarrow$ $B \simeq g: A \rightarrow B$ if for all $h:(A \rightarrow B) \rightarrow I, \Lambda(f) ; h=\perp$ if and only if $\Lambda(g) ; h=\perp$.

Corollary 2. For all $\lambda \nu$ !-terms $M, N: T, M \approx N$ if and only if $\llbracket M \rrbracket \simeq \llbracket N \rrbracket$.

We thus have a full abstraction result for the quotient of the games model under its intrinsic preorder. Note that if $\llbracket M \rrbracket$ and $\llbracket N \rrbracket$ have no globally accessible variables - for example, if $M$ and $N$ are terms of the nu-calculus, or terms over name-free types - then $\llbracket M \rrbracket \simeq \llbracket N \rrbracket$ (and thus $M \approx N$ ) if and only if they have equal sets of complete plays. (A play is complete [2] if the initial question has been answered.) Moreover (unlike the fully abstract model of PCF) observational equivalence of compact strategies is decidable.

Proposition 8. The fully abstract model of $\lambda \nu$ ! is effectively presentable.

Proof. (Sketch) We first show that we can give an effective presentation of the unquotiented model, by giving a finitary representation of compact strategies by restriction to the the legal sequences in which names are introduced in numerical order, starting with zero.

We show that the intrinsic preorder and equivalence on these strategies are decidable by bounding the size of the strategy required to distinguish between given compact strategies. As we have already observed, there are upper bounds $\#(\sigma)$ on the length of sequences in $\sigma$ and $n(\sigma)=\{|\nu(s)| \mid s \in \sigma\}$ on the number of distinct names ocurring in these sequences.

So suppose $\sigma \chi_{\llbracket T \rrbracket} \tau$ - i.e. there exists $\rho: \llbracket T \rrbracket \odot S \rightarrow I$ such that $\sigma ; \rho \neq \tau ; \rho$. We show that there exists $\rho^{\prime}: \llbracket T \rrbracket \odot S \rightarrow I+I$ such that $\sigma ; \rho^{\prime} \neq \tau ; \rho^{\prime}$ and the maximum sequence length in $\rho^{\prime}$ is $\max \{\#(\sigma)$, \# $(\tau)\} \cdot(2 n(\sigma)+2 n(\tau)+1)$. We obtain $\rho^{\prime}$ by eliminating from $\rho$ : 
- sequences which do not arise from interaction with $\sigma$ or $\tau$,

- moves in cells with names which never become globally accessible to $\sigma$ or $\tau$,

- multiple assignments to, or dereferences of the same cell without intervening play in $\llbracket T \rrbracket$.

Thus for any play in $\rho^{\prime}$, the part which is in $\llbracket T \rrbracket \rightarrow I$ is no longer than the maximal play in $\sigma \cup \tau$, whilst the part in the store is at most one dereference of, and assignment to, each name occurring in $\sigma$ or $\tau$ between each pair of moves in $\llbracket T \rrbracket$.

\section{Conclusions and Further Directions}

Several natural questions about our models of local names remain to be answered. For example, which strategies are the denotations of terms of the pure nu-calculus? To characterize them we would need a way of recording the scope of each newly introduced name. It seems unlikely, however, that such a full completeness result would yield an effective characterization of contextual equivalence in the nu-calculus.

There is a more realistic possibility of extending the characterization of observational equivalence in $\lambda \nu$ !, based on our games model. It should, for example, be possible to describe the intrinsic equivalence directly, by characterizing equivalent patterns of behaviour in the store. Recent research 6 19] has succeeded in associating games models of fragments of Idealized Algol with various classes of formal languages, leading to decidability results for observational equivalence. Analogous results may be possible for languages such as $\lambda \nu$ !. However, to characterize a finite-state fragment (for example) it would be necessary to restrict the ability to generate unbounded sets of new names. In general, observational equivalence in $\lambda \nu$ ! is not decidable, because for types generated from $\{o, \rightarrow\}$ it coincides with that of finitary RML, for which undecidability of observational equivalence has been shown by Murawski [17.

As we have noted in the introduction, there are many areas in which local names play a key rôle, and which might therefore be studied semantically using the approach developed here. Most obviously, the games models of higher-order references [4], exceptions [1] and channels [12] could be refined to eliminate bad variables. (A complicating factor in the case of references, for example, is that names are typed.) This might shed light on the categorical structures required to model good variables. We also hope to use the insights into name-passing obtained here to study areas such as mobile and global computation, where it is fundamental. The obvious analogies between names and secrets such as cryptographic keys are suggestive of possible applications in the field of secrecy and security.

\section{References}

1. M. Abadi and A. D. Gordon. A calculus for cryptographic protocols: The spi calculus. Information and Computation, 148(1):1-70, 1999. 
2. S. Abramsky and G. McCusker. Linearity, Sharing and State: a fully abstract game semantics for Idealized Algol with active expressions. In P.W. O'Hearn and R. Tennent, editors, Algol-like languages. Birkhauser, 1997.

3. S. Abramsky and G. McCusker. Call-by-value games. In M. Neilsen and W. Thomas, editors, Proceedings of CSL '97, pages 1-17. Springer-Verlag, 1998.

4. S. Abramsky, K. Honda, G. McCusker. A fully abstract games semantics for general references. In Proceedings of the 13th Annual Symposium on Logic In Computer Science, LICS' '98, 1998.

5. S. Abramsky, R. Jagadeesan and P. Malacaria. Full abstraction for PCF. Information and Computation, 163:409-470, 2000.

6. D. Ghica and G. McCusker. The regular language semantics of second-order Idealised Algol. Theoretical Computer Science, 2003. To appear.

7. K. Honda and N. Yoshida. Game theoretic analysis of call-by-value computation. In Proceedings of ICALP '97, volume 1256 of Lecture Notes in Computer Science. Springer-Verlag, 1997.

8. J. M. E. Hyland and C.-H. L. Ong. On full abstraction for PCF: I, II and III. Information and Computation, 163:285-408, 2000.

9. Alan Jeffrey and Julian Rathke. Towards a theory of bisimulation for local names. In Proceedings of LICS '99. IEEE Press, 1999.

10. J. Laird. A Semantic Analysis of Control. PhD thesis, Department of Computer Science, University of Edinburgh, 1998.

11. J. Laird. A fully abstract game semantics of local exceptions. In Proceedings of LICS '01. IEEE Computer Society Press, 2001.

12. J. Laird. A game semantics of ICSP. In Proceedings of MFPS XVII, number 45 in Electronic notes in Theoretical Computer Science. Elsevier, 2001.

13. O. Laurent. Polarized games. In Proceedings of the Seventeenth International Symposium on Logic In Computer Science, LICS '02, 2002.

14. P. Malacaria and C. Hankin. Non-deterministic games and program analysis: An application to security. In Proceedings of LICS '99, 1999.

15. G. McCusker. Games and full abstraction for a functional metalanguage with recursive types. PhD thesis, Imperial College London, 1996. Published by Cambridge University Press.

16. G. McCusker. On the semantics of the bad-variable constructor in Algol-like languages. In Proceedings of MFPS XIX, ENTCS, 2003. To appear.

17. A. Murawski. On program equivalence in languages with ground-type references. In Proceedings of LICS '03. IEEE Press, 2003.

18. H. Nickau. Hereditarily sequential functionals. In Proceedings of the Symposium on Logical Foundations of Computer Science: Logic at St. Petersburg, LNCS. SpringerVerlag, 1994.

19. C.H.-L. Ong. Observational equivalence of 3rd-order Idealized Algol is decidable. In Proceedings of LICS'02. IEEE Press, 2002.

20. A. Pitts and I. Stark. Observable features of higher-order functions that dynamically create local names. In Proceedings of MFCS '93, pages 122 -141, 1993.

21. J. Power and H. Thielecke. Environments in Freyd categories and $\kappa$-categories. In Proceedings of ICALP '99, number 1644 in LNCS. Springer, 1999.

22. I. Stark. Names and higher-order functions. Technical Report 363, University of Cambridge Computer Laboratory, 1995.

23. I. Stark. Categorical models for local names. Lisp and Symbolic Computation, 1(9):122-141, 1996.

24. E. Sumii and B.C. Pierce. Logical relations for encryption. Journal of Computer Security, 2002. To appear. 